\title{
Gestión del talento humano y rentabilidad del sector hotelero de la región de Puno
}

DOI: https://doi.org/10.21158/01208160.n87.2019.2410

Fecha de recepción: 28 de noviembre de 2018

Fecha de aprobación: 29 de marzo de 2019
Ludwing Roald Flores-Quispe Universidad Privada San Carlos Ludwing.flores@upsc.edu.pe

Cómo citar este artículo / To reference this article / Comment citer cet article / Para citar este artigo:

Flores-Quispe, L. R. (2019). Gestión del talento humano y rentabilidad del sector hotelero de la región de Puno. Revista Escuela de Administración de Negocios, (87), 59-77. DOI: https://doi.org/10.21158/01208160.n87.2019.2410

\section{Resumen}

El artículo tiene como objetivo analizar la incidencia de la gestión del talento humano en la rentabilidad económica y financiera de las organizaciones del sector hotelero, el estudio es de naturaleza cuantitativa y de diseño no experimental, puesto que se analizaron las variables sin la manipulación deliberada, observando los fenómenos tal como se presentan en su contexto natural. Esta investigación se realizó en la región Puno, Perú, dado que la hotelería es una de las principales actividades de esta región.

De esta investigación se concluye que la gestión de personas es un área sensible y prioritaria en las organizaciones del sector hotelero, relevante en el alcance de las metas y objetivos institucionales, constituyéndose así como un eje que repercute y que es altamente significativo respecto a la rentabilidad económica. Se analizaron seis dimensiones de la gestión del talento humano y de ellas las que presentaron la más alta relación respecto a la rentabilidad fueron la admisión y la compensación.

Se evidenció que las empresas del sector hotelero no están siendo lo suficientemente eficientes y se enfrentan al gran reto de la consolidación de sus procesos, aspecto en el que el personal se convierte en el elemento básico de la superación de la empresa, ya que coadyuva a la organización a ganar y mantener una ventaja sostenible. A lo largo del estudio se demostró que el éxito empresarial depende más de la adecuada gestión del talento humano y no tanto de los recursos financieros ni activos fijos como se considera generalmente.

Palabras clave: sector hotelero; talento humano; gestión de talento humano; gestión humana; recursos humanos; rentabilidad económica.

\footnotetext{
${ }^{1}$ Doctor y magíster en Contabilidad y Administración por la Universidad Nacional Del Altiplano. Perú. Contador público por la Universidad Nacional Del Altiplano. Perú. ORCID: https://orcid.org/0000-0002-2946-8710
} 


\section{Human talent management and profitability of the hotel sector in the region of Puno, Perú}

\section{Abstract}

The article aims at analyzing the impact of human talent management on the economic and financial profitability of organizations in the hotel sector. The study is quantitative in nature with a non-experimental design, since the variables were analyzed without deliberate manipulation, observing the phenomena just as they develop in their natural context. This research was carried out in the region of Puno, Peru, as the hotel industry is one of the main activities of this region.

From this research we can conclude that people management is a sensitive area with high priority in the organizations of the hotel sector, relevant in the scope of institutional goals and objectives, thus constituting an axis that has an impact and is highly significant in terms of economic profitability. Six dimensions of human talent management were analyzed, being admission and compensation the ones that presented the highest relationship regarding profitability.

It became apparent that the companies of the hotel sector are not being efficient enough and are facing the great challenge of consolidating their processes, an aspect in which the staff becomes the basic element of overcoming the company, since it helps the organization to gain and maintain a sustainable advantage. Throughout the study, we showed that the success of a business depends more on the proper management of human talent and not so much on financial resources or fixed assets as it is generally considered.

Keywords: hotel sector; human talent; human talent management; human management; human resources; economic profitability.

\section{Gestão de talentos humanos e lucratividade do setor hoteleiro na região de Puno, Peru}

\section{Resumo}

$\mathrm{O}$ artigo tem como objetivo analisar o impacto da gestão de talento humano na rentabilidade econômico-financeira das organizações do setor hoteleiro, o estudo é de natureza quantitativa e de delineamento não experimental, uma vez que as variáveis foram analisadas sem manipulação deliberada, observando os fenômenos como apresentados em seu contexto natural. Esta pesquisa foi realizada na região de Puno, Peru, uma vez que a indústria hoteleira é uma das principais atividades da região.

Esta pesquisa conclui que a gestão de pessoas é uma área sensível e prioritária nas organizações do setor hoteleiro, relevante no âmbito das metas e objetivos institucionais, constituindo assim um eixo que causa impacto e é altamente significativo em termos de rentabilidade econômica. Foram analisadas seis dimensões da gestão de talento humano e, dentre elas, as que apresentaram maior relação em relação à lucratividade foram admissão e remuneração.

Se evidenciou que as empresas do setor hoteleiro não estão sendo suficientemente eficientes e enfrentam o grande desafio de consolidar seus processos, aspecto em que a equipe se torna o elemento básico de superação da empresa, pois ajuda a empresa a obter e manter uma vantagem sustentável. Ao longo do estudo, foi demonstrado que o sucesso nos negócios depende mais do gerenciamento adequado do talento humano e não tanto de recursos financeiros ou ativos fixos como geralmente é considerado.

Palavras-chave: setor hoteleiro; talento humano; gestão de talento humano; gestão humana; recursos humanos; rentabilidade económica 


\section{Gestion des ressources humaines et rentabilité du secteur hôtelier de la région de Puno, au Pérou}

\section{Résumé}

Cet article analyse l'impact de la gestion des ressources humaines sur la rentabilité économique et financière des entreprises du secteur hôtelier. Cette investigation, qui a eu lieu dans la région de Puno, au Pérou, où l'hôtellerie est l'une des principales activités économiques, est de type quantitatif et de conception non expérimentale, et étudie les variables sans manipulation délibérée, en observant les phénomènes en contexte.

Les travaux concluent que la gestion des ressources humaines est une tâche sensible et prioritaire pour les entreprises du secteur hôtelier, tâche à la fois pertinente dans le cadre des objectifs des organisations, et constituant une variable pouvant impacter significativement la rentabilité économique de ces dernières.

Différents facteurs de gestion des ressources humaines ont été analysés. Parmi eux, l'admission et la rémunération des personnels peuvent être considérés comme les facteurs les plus influant sur la rentabilité des entreprises du secteur.

L'étude montre par ailleurs que les entreprises du secteur hôtelier ne sont pas suffisamment efficaces et sont confrontées au défi de la consolidation de leurs processus de gestion, aspect pour lequel le personnel devient un élément clé permettant à l'organisation d'acquérir et de conserver un avantage compétitif durable. Enfin, cette recherche révèle que le succès d'une entreprise dépend davantage d'une gestion efficiente des ressources humaines que des ressources financières ou du capital fixe.

Mots-clés: secteur hôtelier; ressources humaines; gestion des ressources humaines; rentabilité économique. 


\section{Introducción}

$\mathrm{L}$ a gestión del talento humano (GTH) es un área sensible y prioritaria en las organizaciones ya que, en este contexto, se reflejará la eficacia y la rentabilidad esperada (Calderon, 2015). El talento humano es dinámico, sutil y vital, pues depende de aspectos como, por ejemplo, la cultura de cada estructura. Las organizaciones hoteleras poseen las mismas características en cuanto a la gestión de personas, la distribución organizacional adoptada, las características del contexto ambiental, el negocio de la organización, la tecnología utilizada y los procesos internos, pero, sobre todo, al enfocar el talento humano en brindar la seguridad máxima de sus usuarios, ya que es el indicador más importante en empresas con altos riesgos respecto a la integridad y la vida (Correa, 2007; Cabanelas, et al., 2007; Cañas y Millán, 2004; Quintanilla, 2012; Rodríguez y Álvarez, 2011).

La GTH se basa en tres aspectos fundamentales: son sujetos dotados de humanidad que poseen sapiencias, destrezas, técnicas y capacidades indispensables para la gestión adecuada de los recursos organizacionales (Mejía, Rodríguez y Álvarez, 2011). Es el activo más significativo en el sector hotelero, ya que es el personal quien está en contacto directo con los usuarios, desde el personal de ventas hasta los conductores de la organización (Cañas y Millán, 2004; Ortiz, 2013). Las personas son activadores profundos de los recursos empresariales, dado que los individuos son fuentes de propulsión propia que dinamizan la organización, mas no agentes indiferentes, impotentes e inmóviles; son socios en las empresas en estudio, pues invierten en la organización esfuerzo, responsabilidad, compromiso, riesgos, alegrías, amor y vida (Castilla, 2008).

Sin embargo, el nivel de estas características en las empresas del sector hotelero no es tan elevado, ya que el personal, con la expectativa de recibir reintegros de estas inversiones de tiempo - p. ej., salarios, incentivos financieros y crecimiento profesional一, no es retribuido de la misma forma. Cualquier inversión solo se justifica cuando trae un retorno razonable, pero sobre todo humano (Cañas y Millán, 2004).

De acuerdo con la doctrina se tienen los siguientes argumentos respecto a la gestión de personas. Méndez (2015) sostiene que es el proceso de aprovechamiento de las capacidades y habilidades del colaborador de una empresa para lograr los objetivos previstos. Contreras (2006), por su parte, señala que el término «gestión del talento humano» se refiere al conjunto de políticas y prácticas necesarias para dirigir los aspectos de cargos gerenciales relacionados con las personas o los recursos humanos, incluidos el reclutamiento, la selección, la capacitación, las recompensas y la evaluación del desempeño.

Morillo (2001) sostiene que la GTH se refiere a una actividad que depende menos de las escalas, las ordenanzas y las disposiciones, y señala la importancia de una participación activa de todos los colaboradores de la empresa. El objetivo es fomentar una relación de contribución entre los directivos, los gerentes y los colaboradores, a fin de evitar los frecuentes enfrentamientos derivados de una relación diferenciada tradicional (Trias, 2017).

La gestión del talento humano se basa en tres aspectos fundamentales: son seres humanos, ya que están dotados de personalidad y poseen sapiencias, destrezas, habilidades y artes indispensables para la gestión adecuada de los recursos organizacionales; son activadores inteligentes de los recursos organizacionales, puesto que las personas son fuente de impulsos propios que intensifican la estructura, $y$ no agentes pasivos, inactivos y paralizados; además, son afiliados de la organización (Cabanelas, 2007).

Las personas constituyen el principal activo de la organización. Las organizaciones exitosas perciben que solo pueden crecer, prosperar y mantener su continuidad si son capaces de optimizar el retorno sobre las inversiones de todos los socios, en especial de los empleados (Cañas y Millán, 2004; Genesi, 2011). 
Las organizaciones deberían dar mayor importancia al talento humano que poseen y considerar que es un factor primordial para el funcionamiento, el desarrollo y el cumplimiento de los objetivos planteados (Mejía, 2013) ya que el nivel de satisfacción del cliente en la empresa es negativo con relación a la valoración de sus expectativas y el rendimiento percibido.

Según Cantero (2016), el cargo es una unidad de la organización y consta de un continuo de deberes y responsabilidades que lo separan y distinguen de los demás cargos. A través de los cargos la empresa asigna y utiliza los recursos humanos a fin de alcanzar objetivos organizacionales.

Ortiz (2013) plantea que en los nuevos escenarios, por los cuales estamos transitando, se pueden identificar tres aspectos que se destacan por su importancia: la globalización, el permanente cambio del contexto y la valoración del conocimiento (Musa, 2016).La pérdida de capital o de equipamiento posee como vías posibles de solución la cobertura de una prima de seguros o la obtención de un préstamo; no obstante, para la fuga del talento humano estas vías de solución no son posibles de adoptar (Calderón, 2015).

Una empresa se rige por su productividad y competitividad. En razón de que son organizaciones societarias con fines de lucro, es necesario medir su rentabilidad para conservarse en el mercado. Quintanilla (2012) indica que la rentabilidad es una noción que se aplica a toda acción económica en la que se movilizan unos medios materiales, humanos $y$ financieros con el fin de obtener unos resultados.

De acuerdo con lo señalado por Castilla (2008), la rentabilidad financiera o de los fondos propios, denominada en la literatura anglosajona return on equity (ROE), es una medida referida a un determinado periodo de tiempo. Esta información le podrá ser de mucha utilidad ante la solicitud de un préstamo para financiar alguna nueva inversión en la empresa, ya que con este ratio demostraría su capacidad de pago (Correa, 2007).

Finalmente, para la medición objetiva de la rentabilidad es pertinente realizar el cálculo de los ratios financieros. El ratio de liquidez muestra el nivel de solvencia financiera de corto plazo de la empresa, en función de la capacidad que tiene para hacer frente a sus obligaciones de corto plazo que se derivan del ciclo de producción (Cabanelas, 2007). Estos ratios miden la capacidad de pago que tiene la empresa para hacer frente a sus obligaciones en el corto plazo. Es decir, es el dinero en efectivo disponible para cancelar sus deudas, lo cual expresa la capacidad que se tiene de convertir los efectivos en pasivos corrientes (Genesi, 2011; Musa, 2016).

Entre los ratios de liquidez tenemos a la razón corriente, la cual indica el grado de cobertura que tienen los activos de mayor liquidez sobre las obligaciones de menor vencimiento o mayor exigibilidad (Alva, 2009). No obstante, a fin de ser más objetivos se tiene la prueba ácida; esta representa una medida más directa de la solvencia financiera de corto plazo de la empresa, al tomar en consideración los niveles de liquidez de los componentes del activo circulante (Mejía, 2013). Es necesario medir los índices de solvencia que tiene el sector hotelero. Mediante los índices de solvencia medimos la capacidad de endeudamiento de la empresa y su capacidad de pago para enfrentar sus obligaciones o deudas con los medios o recursos que posee (Cañas y Millán, 2004; Musa, 2016), dentro de los cuales se tiende a endeudamiento patrimonial corriente. 


\section{Marco teórico}

T ace poco tiempo que la cultura organizacional 1 predominante en las empresas dejó de privilegiar las tradiciones antiguas y pasadas para concentrarse en el presente. Con esto provocó que el conservadurismo y la preservación del statu quo dieran paso a la innovación y al cambio de hábitos en las maneras de pensar y actuar. La antigua concepción de las relaciones industriales se sustituyó por una nueva manera de administrar el personal, la cual recibió el nombre de «gestión del talento humano». Los departamentos de recursos humanos (DRH) empezaron a percibir a las personas como recursos vivos e inteligentes y ya no como factores inertes de producción.

\subsection{Gestión del talento humano en un ambiente dinámico y competitivo}

Las personas se convierten en el recurso más importante de la organización y en el factor determinante del éxito de la empresa. La tecnología pasó por un increíble e intenso desarrollo, de modo que comenzó a influir en el comportamiento de las organizaciones y de las personas que participaban en ellas. El mundo seguía cambiando. Además, los cambios eran cada vez más veloces (Chiavenato, 2015).
En la era del conocimiento y la información, lidiar con las personas, sus intereses, sus deseos y sus expectativas dejó de ser un problema y se convirtió en una solución para las organizaciones. Dejó de ser un desafío y se convirtió en una ventaja competitiva en las organizaciones con éxito.

\subsection{Las nuevas funciones de la gestión del talento humano}

Busato et al. (2013) comentan que, a fin de crear valor y lograr resultados, un área de recursos humanos debe ubicar no solo las actividades del trabajo a realizar, sino también, las metas y los resultados que le permitan designar las funciones y actividades a las personas de la organización. La figura 1 define las metas junto con las cuatro funciones principales del área. Los ejes vertical y horizontal representan el enfoque en las actividades de recursos humanos. Este enfoque va de lo estratégico a largo plazo a lo operativo de corto. Los profesionales de recursos humanos deben aprender a ser tanto estratégicos como operativos y, de forma simultánea, enfocarse en el largo y corto plazo. Las actividades se extienden de la administración de procesos - herramientas y sistemas de $\mathrm{RH}$ - a la administración del talento humano.

Figura 1. Las funciones de la administración de personas

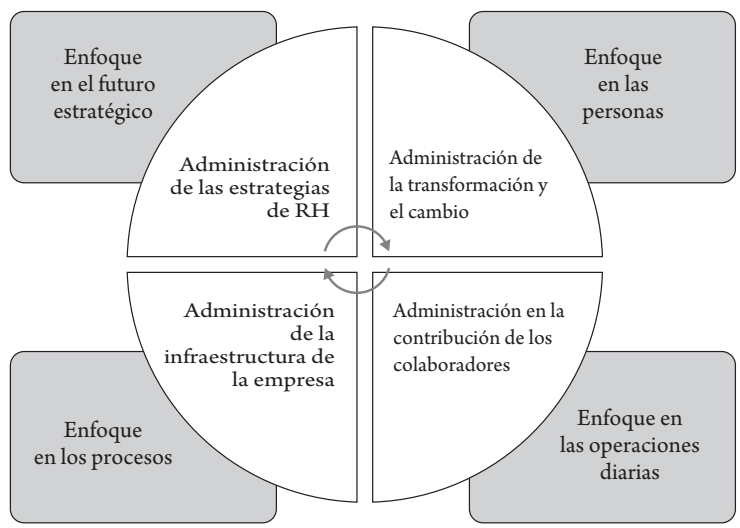

Fuente. Sercap Consulting, 2016, p. 64. 


\subsubsection{Talento humano.}

El concepto de talento humano como eje central de la organización conduce, necesariamente, a la eficacia y la eficiencia. Es el patrimonio incalculable e invaluable que una organización puede reunir en el propósito de alcanzar la competitividad y el éxito empresarial. La administración del talento humano la componen dos aspectos principales:

- Talentos: dotados de conocimientos, sabidurías, destrezas, sentimientos, habilidades y competencias que son reforzados, actualizados y recompensados de forma constante y periódica. Sin embargo, no se puede abordar el talento de forma aislada como un sistema cerrado; solo, no llega lejos, pues debe existir y coexistir en un contexto que le permita libertad, autonomía y cobertura a fin de estar en capacidad de expandirse.
- Contexto: es el ambiente interno adecuado en el que el personal se sienta cómodo y perciba su centro de labores como un lugar al que le apasiona ir, a fin de que los talentos florezcan y crezcan. Sin él, los talentos se marchitan o mueren, por tanto, el contexto es como el hábitat para un ser vivo. El contexto lo determinan aspectos tales como:

» Una arquitectura organizacional con un diseño flexible, integrador y una división del trabajo que coordine a las personas y al flujo de los procesos y de las actividades de manera integral. La organización del trabajo debe facilitar el contacto y la comunicación con las personas.

» Una cultura organizacional democrática y participativa que inspire confianza, compromiso, satisfacción y espíritu de equipo. Una cultura basada en la solidaridad y el amor a lo que se hace.

Figura 2. Componentes de la gestión del talento humano

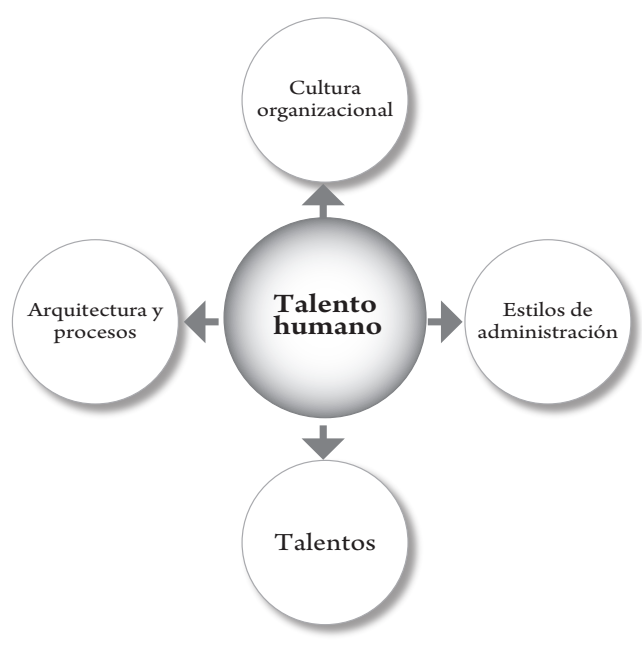

Fuente. Sercap Consulting, 2016, p. 84. 


\subsection{Los seis procesos de la gestión del talento humano}

La gestión del talento humano tiene varias finalidades. Una de ellas es la de retener a las personas que aportan, además de trabajo, profesionalidad a las organizaciones, de modo que le den valor agregado. Este es uno de los comportamientos clave de las organizaciones más inteligentes. Nos sirve también para medir el bienestar de nuestros trabajadores y el clima laboral de la empresa.

El objetivo de la gestión del talento es potenciar este talento innato, así como proporcionar todos los recursos y las herramientas disponibles para su desarrollo.

Los seis procesos de la gestión del talento humano, de acuerdo con la literatura existente, son las siguientes:

- Admisión de personas: es la primera etapa. Comprende el reclutamiento, lo cual forma parte de los procesos dirigidos a incorporar a las personas a la organización. Por medio del reclutamiento, la organización que forma parte del mercado de trabajo (MT) envía una señal a los candidatos, que a su vez forman parte del mercado de recursos humanos (MRH), en la que ofrece oportunidades de empleo. El MT lo componen las ofertas de oportunidades de trabajo que ofrecen las organizaciones, está sujeto a la influencia de varios factores y presenta situaciones que varían de oferta a demanda de empleo. Con la modernización y la globalización, el empleo presenta un perfil nuevo y diferente. El MRH se refiere al conjunto de candidatos a empleo. Se puede segmentar para facilitar la penetración. Tanto el MT como el MRH, que son dos caras de la misma moneda, influyen en el comportamiento de las organizaciones y de las personas (Chiavenato, 2015).

Así mismo, la selección de personal funciona como un filtro que solo permite ingresar a la organización a algunas personas: las más capacitadas y acordes a las competencias de cada puesto en cumplimiento de los objetivos institucionales, y aquellas que cuentan con las características deseadas. Un antiguo concepto popular afirma que la selección consiste en la elección precisa de la persona indicada para el puesto correcto en el momento oportuno. En el fondo, lo que está en juego es el capital intelectual que la organización debe preservar o enriquecer.

- Organización de personas: antes, las organizaciones se conocían por sus predios y por sus cuentas en entidades bancarias. Hoy se conocen por su cultura corporativa. En realidad, la cultura es la forma en que cada organización aprendió a lidiar con su entorno y con sus grupos de interés, trabajadores, clientes, proveedores, accionistas, inversionistas, etc. Es una compleja mezcla de supuestos, creencias, valores, comportamientos, historias, mitos, metáforas y otras ideas que, juntas, representan la forma particular en que funciona y trabaja una organización. Las personas de cada una de esas organizaciones han aprendido una forma peculiar de manejar diversos asuntos relacionados con la vida y el día a día del trabajo (Kido y Kido, 2015).

- Compensación de personas: los procesos dirigidos a recompensar a las personas constituyen los elementos fundamentales para incentivar y motivar a los trabajadores de la organización. De esta forma se mantiene satisfechos y contentos a los colaboradores, quienes se sentirán parte de los beneficios que la organización tienen bajo un principio de igualdad, siempre que los objetivos organizacionales se alcancen y los objetivos individuales sean satisfechos. Por tal razón, los procesos para ofrecer recompensas se destacan entre los principales procesos administrativos del área de recursos humanos dentro de las organizaciones. La palabra «recompensa» significa una «retribución», un «premio»o «reconocimiento» por los servicios de alguien. Una recompensa es un elemento fundamental para conducir a las personas en términos de la retribución, la realimentación o el reconocimiento de su desempeño en la organización (Chiavenato, 2015). 
- Desarrollo de personas: el objetivo de esta fase es mostrar cómo las personas y las organizaciones se desarrollan en razón al aprendizaje continuo y el cambio de comportamiento. Los estratos bajos, como, por ejemplo, la capacitación y el desarrollo de las personas, tratan del aprendizaje a nivel individual y de la manera en que las personas aprenden y se desarrollan. El desarrollo organizacional (DO) es el estrato más amplio y extenso, y se refiere a la manera en que las organizaciones aprenden y se desarrollan gracias al cambio y la innovación (Mizumoto y Lazzarini, 2010).

- Retener a las personas: todos estos procesos pretenden proporcionar un ambiente físico, psicológico y social de trabajo agradable y seguro, así como garantizar relaciones sindicales amigables y cooperativas. Sin embargo, esto suele ser insuficiente si no se tiene por largos periodos a ese personal que tanto costó a la organización motivar, capacitar e, incluso, involucrar en una organización como en una familia, porque se trata de elementos importantes que definen la permanencia de las personas en la organización -y, más que eso, que las motivan a trabajar y a alcanzar los objetivos organizacionales-. En algunas organizaciones los procesos para retener a las personas merecen una calificación baja, porque se acercan al modelo de orden y obediencia a ciegas, el cual hace hincapié en una disciplina rígida, aplicada mediante reglamentos impuestos a las personas con el objeto de estandarizar la disciplina y su desempeño en la organización. Así, el modelo se basa en la estandarización, es decir, se califica a las personas con medias o promedios de parámetros generales y se dejan de lado sus diferencias y características individuales (Rivero y Dabos, 2017).

- Evaluación depersonas: esun procesoquepermite medir el rendimiento laboral del trabajador, si el eje central - que son las personas- ha cumplido con las expectativas empresariales en conformidad con sus actividades, con el objeto de llegar a la toma de decisiones objetivas sobre los recursos humanos.
Las organizaciones modernas utilizan la evaluación del trabajador con el fin de determinar incrementos de sueldos, necesidades de capacitación y desarrollo, así como ofrecer la documentación para apoyar las acciones de rotación de personal.

\subsection{Rentabilidad}

La rentabilidad es una noción que se aplica a toda acción económica en la que se movilizan medios materiales, humanos o financieros con el fin de obtener ciertos resultados. Bajo esta perspectiva, la rentabilidad de una empresa puede evaluarse al comparar el resultado final y el valor de los medios empleados para generar dichos beneficios. Sin embargo, la capacidad de generar las utilidades dependerá de los activos de los que dispone la empresa en la ejecución de sus operaciones, financiados por medio de recursos propios aportados por los accionistas - patrimonio- o por terceros deudas - que implican algún costo de oportunidad, por el principio de la escasez de recursos, y que se toma en cuenta para su evaluación (Bustamante, 2015).

\subsubsection{Tipos de rentabilidad}

Desde el punto de vista contable, el estudio de la rentabilidad se realiza en dos niveles:

- Rentabilidad económica: la rentabilidad económica o de la inversión es una medida del rendimiento de los activos de una empresa con independencia de su financiación, dado en un determinado periodo. Así, esta se constituye como un indicador básico para juzgar la eficiencia empresarial, pues al no considerar las implicancias de financiamiento permite ver qué tan eficiente o viable ha resultado en el ámbito del desarrollo de su actividad económica o gestión productiva. En otros términos, la rentabilidad económica reflejaría la tasa en la que se remunera la totalidad de los recursos utilizados en la explotación. Los ratios más utilizados en su 
medición relacionan cuatro variables de gestión muy importantes para el empresariado: ventas, activos, capital y resultados —utilidades-, como se precisa posteriormente (Roux, 2015).

- Rentabilidad financiera: es una medida referida a un determinado periodo del rendimiento obtenido por los capitales propios, por lo general, con independencia de la distribución del resultado. La rentabilidad financiera puede considerarse así una medida de rentabilidad más cercana a los accionistas o propietarios que la rentabilidad económica, y de ahí que, teóricamente y según la opinión más extendida, sea el indicador de rentabilidad que los directivos buscan maximizar en interés de los propietarios (Bustamante, 2015).

\section{Materiales y métodos}

$\mathrm{E}$ 1 sector hotelero es una actividad a gran escala en la región de Puno, ubicada en la sierra sudeste del país, en la meseta del Collao, a los $13^{\circ} 66^{\prime} 00^{\prime \prime}$ y $17^{\circ} 17^{\prime} 30^{\prime \prime}$ de latitud sur, y los $71^{\circ} 06^{\prime} 57^{\prime \prime}$ y $68^{\circ} 48^{\prime} 46^{\prime \prime}$ de longitud oeste del meridiano de Greenwich. Debido a su ubicación geográfica el espacio físico que comprende, famoso por sus puntos turísticos de renombre mundial, esconde atractivos de singular belleza que poco a poco adquieren valor para la admiración de viajeros nacionales y extranjeros. Allí se puede encontrar magníficos vestigios del pasado, un perfil cultural que aún se conserva después de 2000 años, mil maneras de experimentar la aventura, una gastronomía privilegiada y un folklore muy variado, un lugar de contrastes y gran riqueza natural, cultural y humana. Puno se caracteriza por su gran riqueza cultural. Cuenta con un legado milenario de culturas que se han desarrollado en el pasado y dejaron a futuras generaciones vestigios de su presencia. Entre los vestigios más palpables se encuentran los restos arqueológicos como evidencia clara de un pasado aún presente.

Este trabajo, por su naturaleza de estudio, presenta un enfoque cuantitativo, ya que se usaron las medidas de tendencia central para la medición de la variable independiente gestión del talento humano, y de la misma forma para la variable dependiente rentabilidad. Para estas se aplicaron los ratios financieros en el cálculo de rentabilidad. Al respecto, Hernández et al. (2014) señalan que el enfoque cuantitativo utiliza la recolección y el análisis de datos para contestar preguntas de investigación y probar hipótesis establecidas previamente, y confía en la medición numérica, el conteo y, con frecuencia, en el uso de la estadística, a fin de establecer con exactitud patrones de comportamiento de una población. La investigación es de diseño no experimental, ya que se realiza sin manipular de forma deliberada variables en estudio, de modo que se observan fenómenos tal y como se dan en su contexto natural. En este sentido se estudiaron las variables talento humano y rentabilidad tal como se presentan en la realidad. El nivel de investigación es causal, de manera que el principal objetivo consiste en obtener la evidencia concerniente a las relaciones causales — causa-efecto—. Así, se busca entender la repercusión del talento humano como causa de los resultados económicos. La población la componen las organizaciones hoteleras de 2, 3, 4 y 5 estrellas por su calidad societaria que conforma un total de ciento cinco, de acuerdo con el Ministerio de Comercio Exterior y Turismo. En fin, se aplicó el muestreo estratificado y se obtuvo una muestra de treinta y ocho organizaciones hoteleras. 
Tabla 1. Variables de estudio

\begin{tabular}{|c|c|c|}
\hline Gestión del talen & & Rentabilidad \\
\hline \multicolumn{2}{|l|}{ Dimensiones } & Dimensiones \\
\hline Admisión de personas & $\mathrm{AP}$ & \multirow{6}{*}{$\begin{array}{l}\text { Rentabilidad económica (ROI) } \\
\text { Rentabilidad financiera (ROE) }\end{array}$} \\
\hline Organización de personas & $\mathrm{OP}$ & \\
\hline Compensación de personas & $\mathrm{CP}$ & \\
\hline Desarrollo de personas & $\mathrm{DP}$ & \\
\hline Mantenimiento de personas & MP & \\
\hline Evaluación de personas & $\mathrm{EP}$ & \\
\hline
\end{tabular}

Fuente. Elaboración propia.

\section{Resultados}

$\mathrm{A}^{1}$ 1 tener en cuenta que esta investigación tiene como propósito general comprender de qué manera la gestión del talento humano se convierte en un factor clave para la obtención de resultados económicos favorables en las organizaciones dedicadas al rubro hotelero en la región Puno, desde la visión de los líderes de la gestión humana, Chiavenato (2015) define la gestión del talento humano como el conjunto de habilidades y prácticas necesarias para dirigir los aspectos de los cargos directivos enfocados en las personas o recursos. De esta manera, son seis los procesos de la gestión del talento humano: admisión de personas (AP), organización de personas $(\mathrm{OP})$, compensación de personas $(\mathrm{CP})$, desarrollo de personas (DP), mantenimiento de personas (MP) y evaluación de personas (EP).

Figura 3. Componentes del talento humano

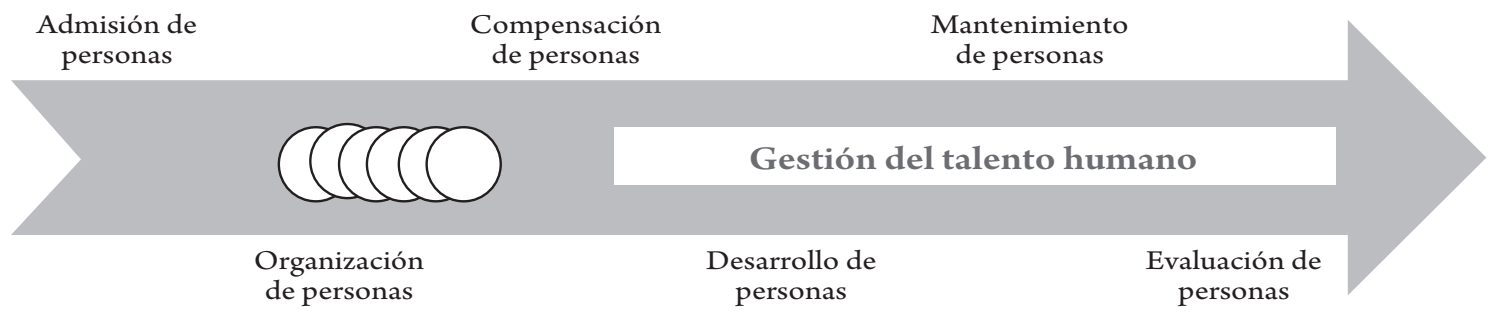

Fuente. Sercap Consulting, 2016, p. 100.

En conformidad con la literatura revisada, la cual enfoca el talento humano como eje central de la organización y su repercusión en los objetivos empresariales, se evaluó cada dimensión de la gestión del talento humano y su repercusión sobre la rentabilidad económica y financiera de las organizaciones en estudio. De esta forma, esta representa una medida de eficiencia y eficacia de las riquezas comprometidas en las organizaciones en estudio, con el fin de garantizar el aumento de valor y su continuidad en el mercado.

Esto significa que no interesa si se generan beneficios muy altos, si para esto se tiene que emplear una considerable cantidad de recursos. Por tanto, una inversión es mejor cuanto mayores son los beneficios que genera y menores son los recursos que requiere 
para obtenerlos. La rentabilidad económica es la medición del rendimiento de los activos de las unidades empresariales hoteleras con independencia de su financiación, dado en un determinado periodo. Así, esta se establece como un indicador básico para juzgar la eficiencia empresarial, pues al no considerar las implicancias de financiamiento permite ver qué tan eficiente o viable ha resultado en el ámbito del desarrollo de su actividad económica o de gestión productiva. En otros términos, la rentabilidad económica refleja la tasa en la que se retribuye la totalidad de los recursos utilizados en la explotación.

Por su parte, la rentabilidad financiera es una medida referida a un determinado periodo del rendimiento obtenido por los capitales propios, por lo general con independencia de la distribución del resultado. La rentabilidad financiera se considera así una medida de rentabilidad más cercana a los accionistas o propietarios que la rentabilidad económica, y de ahí que, teóricamente y según la opinión más extendida, sea el indicador de rentabilidad que los directivos buscan maximizar en interés de los propietarios.

Al utilizar el SPSS 25 para Windows, en el caso de la relación de admisión de personas la rentabilidad económica y la rentabilidad financiera son altamente significativas: $\mathrm{F}=6,818, \mathrm{P}=0,000, \mathrm{y} \mathrm{F}=3,621, \mathrm{P}$ $=0,003$, respectivamente (Tabla 2 ). Los resultados demuestran cómo en las organizaciones que seleccionan de forma adecuada el personal tienen una alta repercusión en los resultados económicos. Las organizaciones escogen a las personas que desean tener como colaboradores y las personas escogen a las organizaciones en las que quieren trabajar y aplicar sus esfuerzos y competencias. Se trata de una elección recíproca que depende de innumerables factores y circunstancias. Sin embargo, a fin de que esa relación sea posible es necesario que las organizaciones comuniquen y divulguen sus oportunidades de trabajo a efecto de que las personas las localicen y puedan iniciar una relación. Esta etapa es determinante en la competitividad de las empresas, por tanto, las organizaciones hoteleras que tienen un riguroso sistema de admisión de personal y orienten estas a su eficiencia son las que obtienen mayores resultados económicos.

En ese sentido, dependerá del enfoque y del objetivo que se propone la organización. Así, encontramos a las empresas del sector hotelero que tienen como objeto buscar a candidatos para ocupar los puestos vacantes dela organizacióny, por otra parte, a las organizaciones que concentran sus esfuerzos de selección en la adquisición de competencias necesarias para el éxito de la organización y aumentar la competitividad del negocio. Cuando el reclutamiento busca atraer candidatos a fin de que ocupen los puestos vacantes y disponibles, vale entonces la pena interesarse en atraer también las competencias necesarias para el éxito de la organización.

Tabla 2. Anova: relación admisión de personas-rentabilidad

\begin{tabular}{|c|l|c|c|c|c|c|}
\hline \multicolumn{2}{|c|}{} & $\begin{array}{c}\text { Suma de } \\
\text { cuadrados }\end{array}$ & Gl & $\begin{array}{c}\text { Media } \\
\text { cuadrática }\end{array}$ & F & Sig. \\
\hline \multirow{3}{*}{ AP-ROI } & Entre grupos & 0,312 & 14 & 0,022 & 6,818 & 0,000 \\
\cline { 2 - 7 } & Dentro de grupos & 0,075 & 23 & 0,003 & & \\
\cline { 2 - 7 } & Total & 0,387 & 37 & & & \\
\hline \multirow{3}{*}{ AP-ROE } & Entre grupos & 0,270 & 14 & 0,019 & 3,621 & 0,003 \\
\cline { 2 - 7 } & Dentro de grupos & 0,123 & 23 & 0,005 & & \\
\cline { 2 - 7 } & Total & 0,393 & 37 & & & \\
\hline
\end{tabular}

Fuente. Elaboración propia. 
En conformidad con la relación de organización de personas y rentabilidad (Tabla 3 ), es altamente significativa $-\mathrm{F}=4,155, \mathrm{P}=0,001$, y $\mathrm{F}=5,075, \mathrm{P}=$ 0,000 - en relación con el ROI y el ROE, respectivamente, al ser un factor clave la organización de las personas si se quiere obtener mejores resultados económicos. En algunas organizaciones dedicadas al sector hotelero estos procesos son básicos, rutinarios e incipientes, ya que se basan en modelos mecanicistas, fundamentados en una visión lógica y determinista —enfocados tan solo en cómo lidiar con las personas-; siguen el modelo burocrático, la repartición del trabajo y la segmentación de las tareas, pese a que le dan importancia a la eficiencia y exigen que las personas desempeñen sus actividades de acuerdo con un método de trabajo preestablecido y monótono que sigue las rutinas y los procedimientos que mandan los directivos.
En un sentido estricto, las personas deben cumplir las reglas impuestas y efectuar sus tareas, asumirlas y no pensar, porque se considera que el método es perfecto, concluido e inmutable. Además, se privilegian los factores higiénicos, porque se concede suma importancia a los aspectos del contexto y a los de la insatisfacción. En estas condiciones, el sistema privilegia el conservadurismo, la rutina y la permanencia de las tareas, dado que nada necesita ni debe cambiarse.

En un mundo que se caracteriza por los intensos cambios y la constante evolución, estos procesos para colocar a las personas se orientan hacia el pasado y no hacia el futuro; estas organizaciones, por ende, tienen una rentabilidad por debajo de la aceptable.

Tabla 3. Anova: relación organización de personas-rentabilidad

\begin{tabular}{|c|l|c|c|c|c|c|}
\hline \multicolumn{2}{|c|}{} & $\begin{array}{c}\text { Suma de } \\
\text { cuadrados }\end{array}$ & Gl & $\begin{array}{c}\text { Media } \\
\text { cuadrática }\end{array}$ & F & Sig. \\
\hline \multirow{3}{*}{ OP-ROI } & Entre grupos & 0,258 & 12 & 0,021 & 4,155 & 0,001 \\
\cline { 2 - 8 } & Dentro de grupos & 0,129 & 25 & 0,005 & & \\
\cline { 2 - 8 } & Total & 0,387 & 37 & & & \\
\hline \multirow{3}{*}{ OP-ROE } & Entre grupos & 0,278 & 12 & 0,023 & 5,075 & 0,000 \\
\cline { 2 - 8 } & Dentro de grupos & 0,114 & 25 & 0,005 & & \\
\cline { 2 - 8 } & Total & 0,393 & 37 & & & \\
\hline
\end{tabular}

Fuente. Elaboración propia.

Sin embargo, se encuentran las empresas que le dan otro enfoque a los procesos dirigidos a colocar a las personas. Estos son más complejos y refinados, porque se basan en modelos orgánicos, fundados en una visión sistémica y con una visión amplia. Siguen el modelo orgánico y son adaptables y flexibles, con alta preponderancia al cambio y a romper paradigmas. Hacen hincapié en la eficacia y exigen que las personas miren más hacia las metas que deben alcanzar y los objetivos que deben cumplir a fin de desempeñar, con cierta libertad de elección, sus actividades, con obediencia de las normas generales de la organización. Así mismo, privilegian los factores motivacionales porque conceden suma importancia a los aspectos del contenido y a los factores de la satisfacción.

En estas condiciones todo es provisional y mutable. El sistema privilegia el cambio, la mejora constante y el desarrollo de las actividades que aseguren la competitividad de la organización. En dichas organizaciones el proceso para colocar a las personas se orienta hacia el futuro y hacia la construcción de su destino. Estas organizaciones tienen los más elevados índices de rentabilidad, y de ahí que se manifiesta una alta relación de significancia entre la organización de personas y los resultados económicos. 
Figura 4. Procesos del talento humano

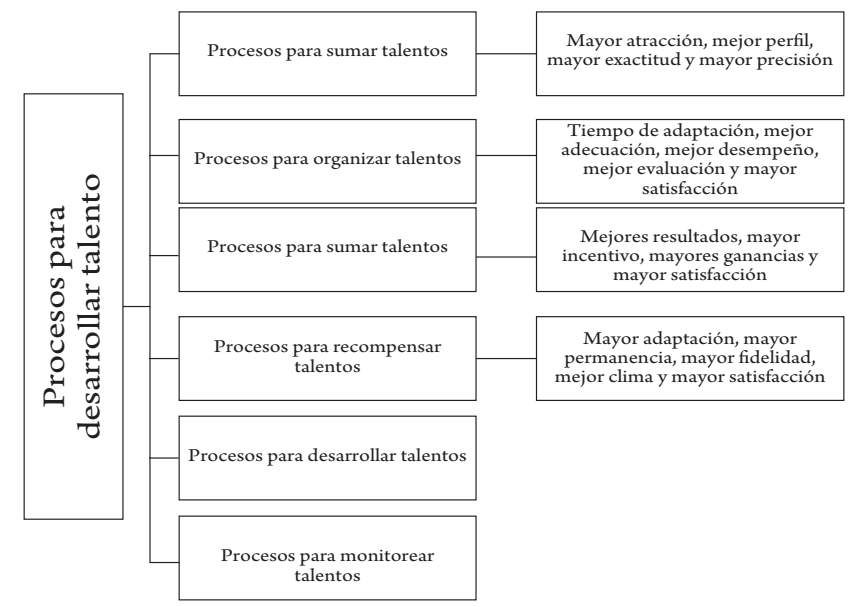

Fuente. Sercap Consulting, 2016, p. 120.

En conformidad con la tabla 4, la relación de compensación de personas y rentabilidad es significativa pese a ser menor que las variables de admisión de personas y organización de personas, y mantiene su importancia respecto al ROI y al ROE $-\mathrm{F}=2,363 ; \mathrm{P}=0,034$ y $\mathrm{F}=2,110 ; \mathrm{F}=0,054$ - respectivamente. Se clasificó la compensación de personas en dos, de manera que es la primera la recompensa financiera y la segunda la recompensa no financiera; para el primer caso el salario, los beneficios sociales, y las bonificaciones; para el segundo, las oportunidades de desarrollo, el reconocimiento y la autoestima, la seguridad, la calidad de vida, la promociones, la libertad y la autonomía en el trabajo.

Tabla 4. Anova: relación compensación de personas-rentabilidad

\begin{tabular}{|c|l|c|c|c|c|c|}
\hline \multicolumn{2}{|c|}{} & $\begin{array}{c}\text { Suma de } \\
\text { cuadrados }\end{array}$ & Gl & $\begin{array}{c}\text { Media } \\
\text { cuadrática }\end{array}$ & F & Sig. \\
\hline \multirow{4}{*}{ CP-ROI } & Entre grupos & 0,206 & 12 & 0,017 & 2,363 & 0,034 \\
\cline { 2 - 7 } & Dentro de grupos & 0,181 & 25 & 0,007 & & \\
\cline { 2 - 7 } & Total & 0,387 & 37 & & & \\
\hline \multirow{3}{*}{ CP-ROE } & Entre grupos & 0,198 & 12 & 0,016 & 2,110 & 0,054 \\
\cline { 2 - 7 } & Dentro de grupos & 0,195 & 25 & 0,008 & & \\
\cline { 2 - 7 } & Total & 0,393 & 37 & & & \\
\hline
\end{tabular}

Fuente. Elaboración propia.

Las organizaciones hoteleras, en muchos de los casos, no tienen sistemas de recompensas para sus miembros. Una recompensa es una retribución, un estímulo o un reconocimiento por los servicios de los colaboradores. La recompensa más común es la remuneración. La remuneración total es el paquete de recompensas cuantificables que el ser humano recibe por su trabajo y lo componen la remuneración básica, los beneficios sociales, los incentivos salariales y las prestaciones. Estas últimas, por ende, tienen un rendimiento económico por debajo del promedio y aceptable dentro del rubro hotelero, ya que la remuneración es el proceso que involucra todas las formas de pago o de recompensas que se entregan 
a los trabajadores y se derivan de su empleo. En las organizaciones hoteleras se observó que el salario se puede tasar en una unidad de tiempo - mes u hora de trabajo-, en los resultados o en la tarea.

El salario es importante para la persona —-define su nivel de vida - y para la organización —impacta en sus costos-, y depende de factores internos organizacionales-y externos - ambientales-. Sin embargo, si no se cuida este aspecto no se conseguirán los objetivos empresariales. De la misma forma, las organizaciones que cuidan este aspecto mantienen una mejor posición con respecto a los indicadores económicos, al ser muy estrecha la relación entre la compensación de personas y la rentabilidad.

Como se observa en la tabla 5, se tiene una significativa relación entre el desarrollo de personas con la rentabilidad económica y financiera. Los resultados $\mathrm{F}=4,434, \mathrm{P}=0,001$, y $\mathrm{F}=2,815, \mathrm{P}=$ 0,016 , respectivamente, nos indican que existe relación lineal entre las variables analizadas y que tiene sentido el análisis aplicado al modelo planteado. Se debe precisar que las personas tienen una increíble capacidad de aprendery desarrollarse, de modo que la educación se encuentra en el centro de esa capacidad.
Los procesos de desarrollo de las personas tienen una estrecha relación con la rentabilidad, representa la necesidad que tiene el ser humano de llevar de adentro hacia fuera sus potenciales internos; significa exteriorizar ese estado latente y el talento creador e innovador de las personas. Cualquier modelo de formación, capacitación, educación, entrenamiento o desarrollo debe garantizar la posibilidad de realizar todo aquello que el hombre puede ser en razón de sus propios potenciales, bien sean innatos o bien adquiridos.

Chiavenato (2015) manifiesta que el cambio es el factor que más afecta las actividades de capacitación y desarrollo. Sin embargo, otros factores que afectan la posibilidad de alcanzar estos objetivos en las organizaciones son: el apoyo de la alta gerencia, el compromiso de los especialistas, los avances tecnológicos y los principios del aprendizaje. En las organizaciones hoteleras las empresas que cuentan con el nulo apoyo de la alta gerencia hacia la capacitación son las que tienen los indicadores de rentabilidad más bajos; de forma paralela, las organizaciones que mayor importancia le dan a este indicador son las que obtienen rendimientos económicos positivos.

Tabla 5. Anova: relación desarrollo de personas-rentabilidad

\begin{tabular}{|c|l|c|c|c|c|c|}
\hline \multicolumn{2}{|c|}{} & $\begin{array}{c}\text { Suma de } \\
\text { cuadrados }\end{array}$ & gl & $\begin{array}{c}\text { Media } \\
\text { cuadrática }\end{array}$ & F & Sig. \\
\hline \multirow{3}{*}{ DP- ROI } & Entre grupos & 0,240 & 10 & 0,024 & 4,434 & 0,001 \\
\cline { 2 - 7 } & Dentro de grupos & 0,146 & 27 & 0,005 & & \\
\cline { 2 - 8 } & Total & 0,387 & 37 & & & \\
\hline \multirow{3}{*}{ DP- ROE } & Entre grupos & 0,200 & 10 & 0,020 & 2,815 & 0,016 \\
\cline { 2 - 8 } & Dentro de grupos & 0,192 & 27 & 0,007 & & \\
\cline { 2 - 8 } & Total & 0,393 & 37 & & & \\
\hline
\end{tabular}

Fuente. Elaboración propia.

Uno de los componentes básicos de la gestión del talento humano es la retención de personas, ya que la organización viable es aquella que no solo consigue captar y aplicar de forma conveniente sus recursos humanos, sino que también los mantiene satisfechos, motivados, alegres y conformes con su centro de labor a largo plazo dentro de la organización. La retención de las personas exige poner atención especial a un conjunto de cuestiones, entre las cuales sobresalen los estilos administrativos, las relaciones con los empleados, la confianza hacia ellos, el trato, el respeto a su dignidad y los programas de higiene y seguridad 
en el trabajo que aseguran la calidad de vida dentro de la organización. Los procesos dirigidos a retener a las personas tienen por objeto mantener a los participantes satisfechos y motivados, así como asegurarles las condiciones físicas, psicológicas y sociales para que permanezcan en la organización, se comprometan con ella $\mathrm{y} \ll$ se pongan la camiseta».

Tabla 6. Anova: relación mantenimiento de personas-rentabilidad

\begin{tabular}{|c|l|c|c|c|c|c|}
\hline \multicolumn{2}{|c|}{} & $\begin{array}{c}\text { Suma de } \\
\text { cuadrados }\end{array}$ & Gl & $\begin{array}{c}\text { Media } \\
\text { cuadrática }\end{array}$ & F & Sig. \\
\hline \multirow{3}{*}{ MP-ROI } & Entre grupos & 0,269 & 10 & 0,027 & 6,149 & 0,000 \\
\cline { 2 - 7 } & Dentro de grupos & 0,118 & 27 & 0,004 & & \\
\cline { 2 - 7 } & Total & 0,387 & 37 & & & \\
\hline \multirow{3}{*}{ MP-ROE } & Entre grupos & 0,171 & 10 & 0,017 & 2,080 & 0,064 \\
\cline { 2 - 7 } & Dentro de grupos & 0,222 & 27 & 0,008 & & \\
\cline { 2 - 7 } & Total & 0,393 & 37 & & & \\
\hline
\end{tabular}

Fuente. Elaboración propia.

Según los resultados obtenidos, como se observan en la tabla 6, existe una relación altamente significativa entre el mantenimiento de personas con la rentabilidad obtenida, al obtenerse para el ROI y el ROE F =6,149, P = 0,000, y F $=2.080, \mathrm{P}=0,064$, respectivamente. Así, es mayor la relación de significatividad con la rentabilidad económica respecto a la rentabilidad financiera.

Tabla 7. Anova: relación evaluación de personas-rentabilidad

\begin{tabular}{|c|l|c|c|c|c|c|}
\hline \multicolumn{2}{|c|}{} & $\begin{array}{c}\text { Suma de } \\
\text { cuadrados }\end{array}$ & Gl & $\begin{array}{c}\text { Media } \\
\text { cuadrática }\end{array}$ & F & Sig. \\
\hline \multirow{3}{*}{ EP-ROI } & Entre grupos & 0,195 & 11 & 0,018 & 2,337 & 0,037 \\
\cline { 2 - 7 } & Dentro de grupos & 0,198 & 26 & 0,008 & & \\
\cline { 2 - 7 } & Total & 0,393 & 37 & & & \\
\hline \multirow{3}{*}{ EP-ROE } & Entre grupos & 0,193 & 11 & 0,018 & 2,317 & 0,038 \\
\cline { 2 - 7 } & Dentro de grupos & 0,197 & 26 & 0,008 & & \\
\cline { 2 - 7 } & Total & 0,391 & 37 & & & \\
\hline
\end{tabular}

Fuente. Elaboración propia.

Las organizaciones no operan en razón a la simple improvisación ni funcionan por azar, y si lo hacen tienden a perder la eficacia de sus funciones $y$ actividades como tal. Las organizaciones se rigen por determinados planes que tienen por objeto alcanzar objetivos, cumplir la misión y realizar la visión por medio de estrategias bien definidas. El comportamiento organizacional no puede ser imprevisto ni errático, sino que debe ser deliberado, empático y racional. Por lo mismo, las organizaciones deben hacer un esfuerzo considerable para supervisar sus diversas operaciones y actividades.

Como se observa en la tabla 7, entre la relación de evaluación de personas con la rentabilidad económica y financiera de $\mathrm{F}=2,337, \mathrm{P}=0,037, \mathrm{y} \mathrm{F}=2,317, \mathrm{P}=$ 0,038 , respectivamente, si bien es cierto la relación 
es menor a las variables anteriores, no por eso se debe pasar por alto, ya que supervisar significa hacer seguimiento a esas operaciones y actividades con el fin de garantizar que los planes sean debidamente ejecutados y los objetivos se alcancen. Los procesos de supervisión se aplican a las funciones que permiten alcanzar los objetivos en razón a la actividad de las personas que componen la organización. En este sentido, la función de staff del departamento de recursos humanos y la responsabilidad general de línea tienen tareas precisas. La función de las empresas hoteleras es diseñar sistemas que permitan reunir y obtener los datos que abastecerán al sistema de información administrativa que sentará las bases para las decisiones de la gerencia de línea. Los gerentes y los colaboradores deben tener información en tiempo real a fin de tomar decisiones y hacer que sucedan las cosas, y de tal modo obtener los beneficios esperados.

Tabla 8. Prueba de normalidad

\begin{tabular}{|c|c|c|c|c|}
\hline \multicolumn{5}{|c|}{ Shapiro-Wilk } \\
\hline \multirow{4}{*}{ Rentabilidad } & Variables dependientes & Estadístico & gl & Sig. \\
\cline { 2 - 5 } & AP & 0,933 & 38 & 0,005 \\
\cline { 2 - 5 } & OP & 0,936 & 38 & 0,010 \\
\cline { 2 - 5 } & CP & 0,939 & 38 & 0,011 \\
\cline { 2 - 5 } & DP & 0,905 & 38 & 0,003 \\
\cline { 2 - 5 } & MP & 0,976 & 38 & 0,045 \\
\hline
\end{tabular}

Fuente. Elaboración propia.

Conforme a la tabla 8 , se tiene que todas las variables dependientes presentan una distribución normal respecto a la misma varianza, lo cual les otorga mayor solidez a los cuadros precedentes. En razón a estos resultados, se proporciona una ganancia mayor sobre las aportaciones efectuadas por los grupos de interés y se mantiene la continuidad del negocio. Las organizaciones procuran privilegiar a los grupos de interés más importantes. Accionistas e inversionistas eran, hasta hace poco tiempo, los más privilegiados en la distribución y la apropiación de los resultados de la organización. Esa asimetría se sustituye de manera progresiva por una visión sistémica e integral de todos los grupos de interés del negocio. Esto se ha demostrado mediante las pruebas de correlación entre la GTH y la rentabilidad, ya que todos ellos son indispensables para el éxito de la empresa y, por tanto, resulta que el asociado más íntimo y más importante de la organización es el colaborador, aquel que está dentro de ella, quien le da vida, amor y dinamismo y hace que sucedan las cosas de acuerdo con los objetivos plasmados. 


\section{Conclusiones}

T a gestión de personas es un área sensible y Uprioritaria en las organizaciones del sector hotelero, predominante en lo que se refiere a las metas y objetivos institucionales. Se constituye como el eje central, ya que repercute y es altamente significativa en cuanto a la rentabilidad económica. De las seis dimensiones de la gestión del talento humano en estudio, las que mantienen la más alta relación respecto a la rentabilidad son la admisión y la compensación de personas.

De las dimensiones de la gestión del talento humano y su relación con la rentabilidad financiera las que cobran mayor significatividad son la organización y la admisión de personas, y es preponderante el adecuado reclutamiento y selección de personal esta es el cimiento de las organizaciones hoteleras, y de ella dependerá el futuro de la organización-.
Estos resultados se convierten en una guía para las organizaciones del sector hotelero de la región Puno que deseen mejorar sus rendimientos económicos, de modo que deberán centrar su atención en la adecuada gestión de sus colaboradores desde la admisión de personas, al ser esta la base y el eje fundamental para el desarrollo organizacional.

Las empresas del sector hotelero no son lo suficientemente eficientes y se enfrentan con un gran reto como es la consolidación de sus procesos. Estas organizaciones no superan las expectativas de su rentabilidad tanto económica como financiera, de modo que las personas se convierten en el elemento básico de la superación de la empresa que coadyuva a la organización a ganar y mantener una ventaja sostenible. El estudio demuestra que el éxito empresarial depende de la adecuada gestión del talento humano y no de los recursos financieros ni de los activos fijos, como se creyó durante mucho tiempo.

\section{Referencias}

Alva, J. (2009). Rentabilidad y eficiencia de las entidades financieras de economía social en España. Revista Venezolana de Gerencia (RVG), 14(45), 24-41. DOI: https://doi. org/10.31876/revista.v14i45.10520

Busato, A.; Santos, D.; Freddo, C. (2013). Representações sociais dos acadêmicos de Administração sobre recursos humanos. Revista de Gestão, 20(4), 461-476. DOI: https:// doi.org/10.5700/rege512

Bustamante, C. (2015). Análisis de rentabilidad de una empresa (2a. ed.). Lima: Editores-hoyli.

Cabanelas, P. (2007). Reclutamiento de personal y sus valores. Revista Galega de Economía, 16(1), 1-19.

Calderón, L. (2015). El talento humano como ventaja competitiva. Revista Galega de Economía, 89(3), 223-259.

Cañas, E.; Millán, I. (2004). Artículo 2. Suma de Negocios, 21(2), 581-584.
Cantero, H. (2016). La gestión del talento humano orientado a las nuevas tecnologías de información. Ciencias Holguín, 22(4), 1-17. Recuperado de http://www.redalyc.org/ articulo.oa?id=181548029004

Castilla, R. (2008). El talento humano como factor clave del éxito empresarial. Revista de Ciencias de la Administración y Economía, 65(2), 120-125.

Chiavenato, I. (2015). Gestión del talento humano. Instituto Tecnológico y de Estudios Superiores de Monterrey, Campus Ciudad de México, Departamento Académico de Administración.

Contreras, I. (2006). Las relaciones laborales como factor de productividad. Visión Gerencial, 7(7), 1-7.

Correa, J. (2007). Las organizaciones del siglo XXI. Revista Escuela de Administración de Negocios, 60(5),47-164. DOI: https://doi.org/10.1590/S0100 
Genesi, R. (2011). El capital humano y su nuevo enfoque empresarial. Negotium, 6(18),128.

Hernández R.; Fernández, C.; Baptista, P. (2014). Metodología de la investigación (4a. ed.). México D. F.: McGraw Hill.

Kido, A.; Kido, T. (2015). Modelos teóricos del capital humano y señalización: un estudio para México. Contaduría y Administración, 60(4), 723-734. DOI: https://doi. org/10.1016/j.cya.2014.06.001

Mejía, G. (2013). La gestión del talento humano y su relación con las nuevas tecnologías. Ingeniería Industrial, XXXIV(1), 2-11. Recuperado de http://www.redalyc.org/articulo. oa? id $=360433593002$

Méndez, M. (2015). Recursos humanos y productividad. Revista Venezolana de Sociología y Antropología Fermentum, 45(12), 22. Recuperado de http://www.redalyc.org/articulo. oa? id=70504404

Mizumoto, F.; Lazzarini, S. (2010). Humano, capital social e práticas gerenciais. Revista de Administração, 45(4), 343-355. DOI: https://doi.org/10.1016/S0080-2107(16)30466-6

Morillo, M. (2001). Administración de recursos humanos y sostenibilidad. Venezuela: Universidad de Los Andes.

Musa, M. (2016). Los colaboradores y su importancia en la rentabilidad de las empresas de servicios en Málaga. Revista de Investigación, 45(88). Recuperado de https://bit.ly/3dpvOJy

Ortiz, M. (2013). Desempeño logístico y rentabilidad económica. Fundamentos teóricos y resultados prácticos. Economía y Desarrollo, 149(5), 182-193. Recuperado de http://www.redalyc.org/articulo.oa?id=425541207012

Quintanilla, J. (2012). Gestión del talento humano y la calidad en corporaciones de manufactura. Revista de Ciencias de La Administración y Economía, 4(3), 105-114. Recuperado de http://www.redalyc.org/pdf/5045/504550953007.pdf

Rivero, A.; Dabos, G. (2017). Gestión diferencial de recursos humanos: una revisión e integración de la literatura. Estudios Gerenciales, 33(142), 39-51.DOI: https://doi.org/10.1016/j. estger.2016.12.003

Rodríguez, F.; Álvarez, C. (2011). El talento humano y la innovación empresarial en el contexto de las redes empresariales: el clúster de prendas de vestir en Caldas-Colombia. Estudios Gerenciales, 27(119), 209-232. DOI: https://doi.org/10.1016/S0123-5923(11)70164-4

Roux, A. (2015). Medidas de desempenho da area de recursos humanos e seu relacionamento com indicadores de desempenho econômico. REGE-Revista de Gestão, 22(1), 97-114. Recuperado de https://www.revistas.usp.br/rege/ article/view/102626

Trias, E. (2017). Gestión del talento humano. Contabilidad y Administración, 2(3), 1-35. 
